$\mathrm{CDF} / \mathrm{DOC} / \mathrm{CDF} / \mathrm{CDFR} / 10650$

FERMILAB-FN-0929

\title{
$p \bar{p}$ cross sections for the Tevatron energy scan
}

\author{
Nathan Goldschmidt
}

September 7, 2011

\begin{abstract}
The Tevatron energy scan will present unique operational challenges for the CDF detector as a whole and the CLC luminosity detector in particular. The primary concern for CLC operations is the energy dependence of the $p \bar{p}$ cross section. In this note, values for the hadronic $p \bar{p}$ cross section at the center-of-mass energies to be visited during the scan are tabulated.
\end{abstract}


The CLC [1] measures $\mu$, the average number of interactions per bunch crossing in $p \bar{p}$ collisions at the Tevatron. The relationship between $\mu$ and the total rate of $p \bar{p}$ collisions is,

$$
\mathcal{L}=\frac{\mu \cdot f_{\mathrm{BC}}}{\sigma_{\text {tot. }}}
$$

where rate of bunch crossings, $f_{\mathrm{BC}}$, is $48 \mathrm{kHz}$ and $\sigma_{\text {tot. }}$ is the total $p \bar{p}$ cross section. At $\sqrt{s}=1.96 \mathrm{TeV}$ the total $p \bar{p}$ cross section is $81.90 \pm 2.30 \mathrm{mb}[2,3]$. The CLC is insensitive to elastic scattering - the inelastic $p \bar{p}$ cross section used in the luminosity calculation for collisions at $\sqrt{s}=1.96 \mathrm{TeV}$ is $\sigma_{\text {inel. }}=$ $61.9 \pm 1.4 \mathrm{mb}[3]$.

The Tevatron energy scan will collide protons and antiprotons at centerof-mass energies of 300,630 , and $900 \mathrm{GeV}$. The total, elastic and inelastic cross sections at these energies are determined according to data provided by the Particle Data Group [6,7]. At each center-of-mass energy to be visited during the scan, the cross section is estimated by performing a linear interpolation between the adjacent data points. These cross sections are given in Table 1. Reference is given to the original publications for data used in the interpolation.

It is worth noting that the acceptance for events to be measured by the CLC will differ for varying center-of-mass energies. To estimate the acceptance, a study including full simulation of the detector elements is required. Unfortunately, such a study has not been preformed at these energies. The acceptance can be estimated a posteriori by examining zero-bias data and applying the method described in CDF note 6054 [5].

\section{References}

[1] D. Acosta et al. "The CDF Cherenkov luminosity monitor," Nucl. Instrum. Meth. A 461, 540 FERMILAB-PUB-01/031-E (2001).

[2] S. Klimenko, J. Konigsberg, and T. Liss, "Average cross-section of the inelastic $p \bar{p}$ scattering at 1.8 TeV" CDF internal note CDF/DOC/CDF/CDFR/6314 (2003).

[3] J. Konigsberg et al. "A first look at the CLC luminosity measurements," CDF internal note CDF/ANAL/CDF/CDFR/6052 (2002). 

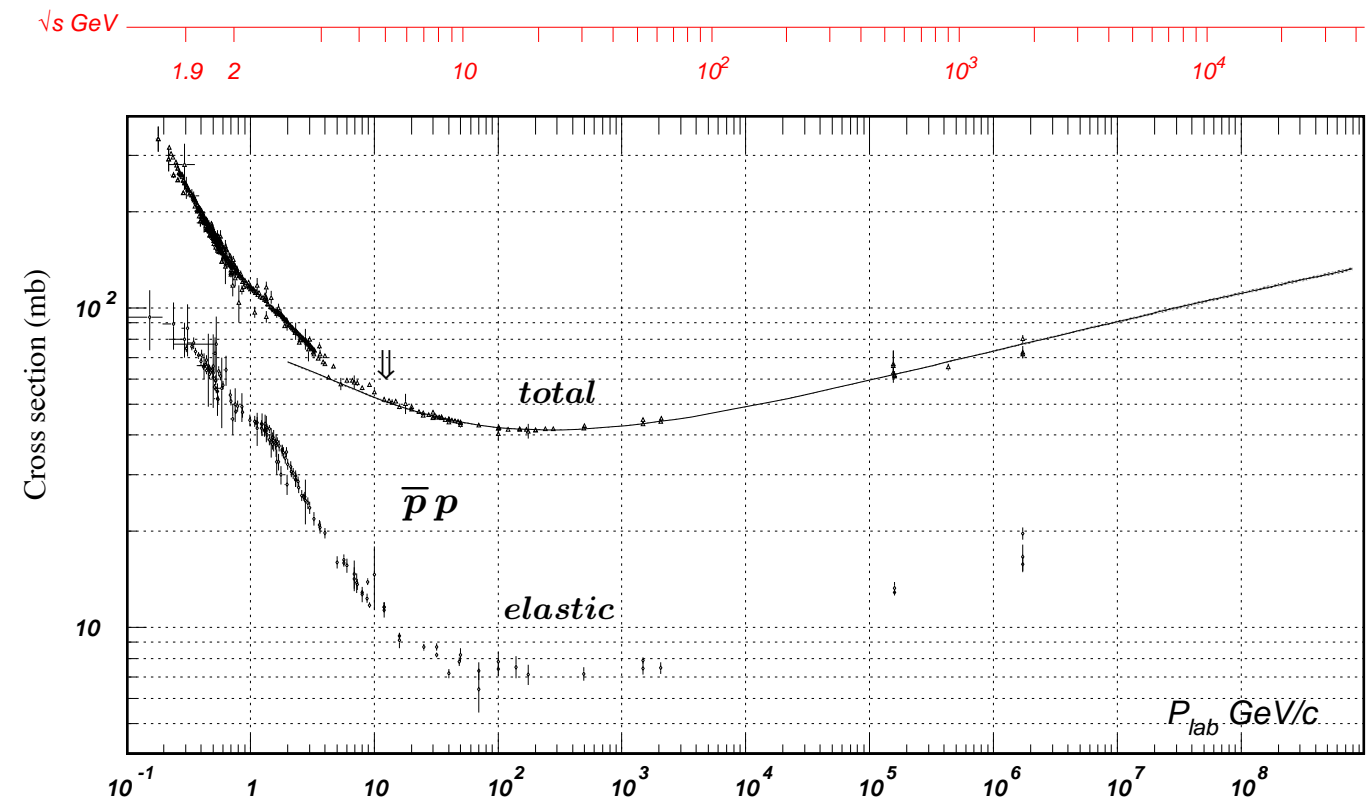

Figure 1: Total and elastic cross sections for and $p \bar{p}$ collisions as a function of laboratory beam momentum and total center-of-mass energy. Corresponding computer-readable data files may be found at http://pdg.lbl.gov/2011/hadronicxsections/. (Courtesy of the COMPAS group, IHEP, Protvino, August 2005) [6,7].

[4] S. Jindariani et al. "Luminosity uncertainty for run 2 up until August 2004," CDF internal note CDF/ANAL/CDF/7446 (2005).

[5] D. Acosta et al. "Estimation of CLC acceptance to inelastic p-pbar interactions," CDF internal note CDF/DOC/CDF/CDFR/6054 (2002).

[6] K. Nakamura et al. (Particle Data Group), J. Phys. G 37, 075021 (2010).

[7] http://pdg.lbl.gov/2011/hadronic-xsections/

[8] D. V. Bugg et al. " $\bar{p} p$ total cross sections below $420 \mathrm{MeV} / c$," Phys. Lett. B 194, 563 (1987).

[9] V. Chaloupka et al. (CERN-Liverpool-Mons-Padua-Rome-Trieste Collaboration), "Measurement of the total and partial anti-p p cross-section between $1901 \mathrm{MeV}$ and $1950 \mathrm{MeV}, "$ Phys. Lett. B 61, 487 (1976).

[10] R. P. Hamilton et al. "Measurement of the $\bar{p} p$ Charge-Exchange Cross Section from 0.119 to $1.046 \mathrm{GeV} / c$," Phys. Rev. Lett. 44, 1179 (1980). 


\begin{tabular}{r|c|c|c}
\hline \hline$\sqrt{s}$ & $300 \mathrm{GeV}$ & $630 \mathrm{GeV}$ & $900 \mathrm{GeV}$ \\
\hline$\sigma_{\text {tot. }}(\mathrm{mb})$ & $51.6 \pm 1.4 \pm 0.9$ & $62.5 \pm 3.5 \pm 1.1$ & $65.3 \pm 0.7 \pm 2.3$ \\
$\sigma_{\text {el. }}(\mathrm{mb})$ & $9.0 \pm 11.4 \pm 0.0$ & $13.4 \pm 1.5 \pm 0.0$ & $13.7 \pm 1.4 \pm 0.0$ \\
$\sigma_{\text {inel. }}(\mathrm{mb})$ & $42.6 \pm 11.5 \pm 0.9$ & $49.2 \pm 3.8 \pm 1.1$ & $51.6 \pm 1.6 \pm 2.3$ \\
\hline \hline
\end{tabular}

Table 1: Total, inelastic and elastic cross sections for $p \bar{p}$ scattering at $\sqrt{s}=300$, 630 , and $900 \mathrm{GeV}$, according to [6,7]. The first error is statistical, the second systematic.

[11] A. S. Clough et al. "Evidence against the S-meson," Phys. Lett. B 146, 299 (1984).

[12] G. J. Alner et al. (UA5 Collaboration), "Antiproton-proton cross sections at 200 and 900 GeV c.m. energy," Z. Phys. C 32, 153 (1986). 


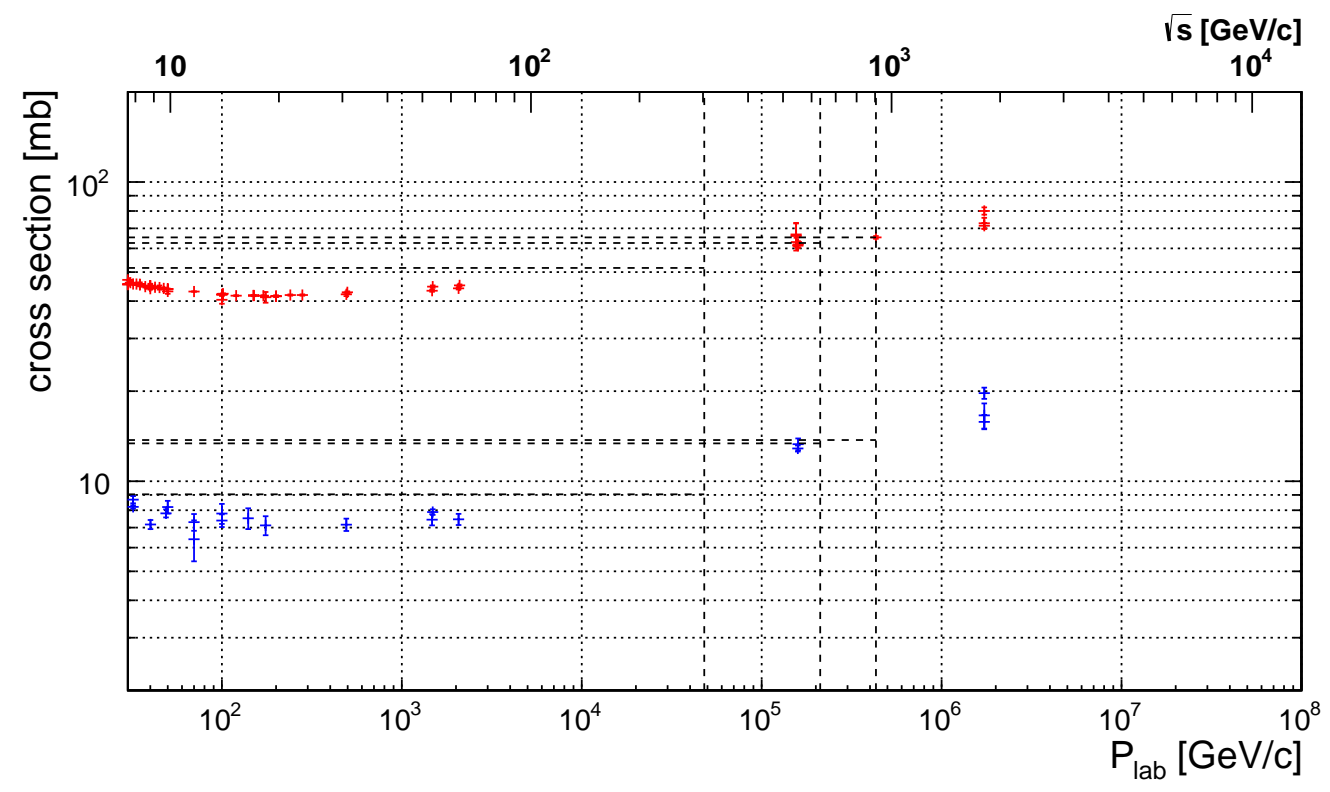

Figure 2: Total and elastic cross sections for and $p \bar{p}$ collisions as a function of laboratory beam momentum and total center-of-mass energy. The red points indicate the total cross section, the blue points the elastic cross section. The center-of-mass energies to be visited during the Tevatron energy scan $(\sqrt{s}=$ 300, 630, $900 \mathrm{GeV}$ ) are indicated by dashed lines. [6,7]. 